\title{
PAPER \\ A Novel Modulation with Parallel Combinatory and High Compaction Multi-Carrier Modulation
}

Yafei HOU ${ }^{\dagger}$ and Masanori HAMAMURA ${ }^{\dagger \dagger \mathrm{a})}$, Members

SUMMARY In this paper, we propose a new modulation named parallel combinatory/high compaction multi-carrier modulation (PC/HC-MCM) using the techniques of parallel combinatory orthogonal frequency division multiplexing (PC-OFDM) and high compaction multi-carrier modulation (HC-MCM). Two types of PC/HC-MCM systems, which are named as modulated PC/HC-MCM system and (unmodulated) PC/HC-MCM system, can be designed. The modulated PC/HC-MCM system achieves better bit-error rate (BER) performance than that of HC-MCM system with equal bandwidth efficiency (BWE). The PC/HC-MCM system can obtain the better peak-to-average power ratio (PAPR) characteristics by selecting appropriate constellation for each subcarrier. On the other hand, since PC/HC-MCM can divide the PC-OFDM symbol duration into multiple time-slots, the advantages of frequency hopping $(\mathrm{FH})$ can be applied in the PC/HC-MCM system. Therefore, we also combine the PC/HC-MCM and frequency hopping multiple access (FHMA) to propose a novel multiple access (MA) system. It can simultaneously transmit multiple users' data within one symbol duration of PC-OFDM.

key words: PC-OFDM, HC-MCM, bandwidth efficiency, multiple access, frequency hopping, PAPR reduction

\section{Introduction}

Orthogonal frequency division multiplexing (OFDM) is a well-known multi-carrier transmission system which has been attracted by its high tolerance to multipath interference in wireless networks [1]. So OFDM systems have been selected as the standards for digital video broadcasting (DVB) and digital audio broadcasting (DAB). It achieves high bandwidth efficiency for applications including wireless multimedia, wireless internet access, future generation mobile communication systems and power-line communication systems [2], [3].

In the OFDM, serial data stream is first converted to parallel and then parallel data are transmitted by subcarriers synchronously. Parallel transmission method including the OFDM can divide the high rate signal into sub-flows with lower rate, therefore, can mitigate the influence due to multipath. One of the advantages for the use of the parallel combinatory method is that the transmission with the parallel combinatory coding is available in addition to the parallel

Manuscript received August 30, 2005.

Manuscript revised November 20, 2006.

Final manuscript received August 15, 2007.

${ }^{\dagger}$ The author is with the Graduate School of Engineering, Kochi University of Technology, Japan and Dept. of Computing and Information Technology, Fudan University, P.R. China.

${ }^{\dagger}$ The author is with the Dept. of Information Systems Engineering, Kochi University of Technology, Kami-shi, 782-8502 Japan.

a) E-mail: hamamura.masanori@kochi-tech.ac.jp DOI: 10.1093/ietfec/e90-a.11.2556 data transmission [4], [5]. The system selects some subcarriers and transmits the special signals according to the combinatory rule, for example, only zero amplitude point, in the receiver, the system recovers the additional bits by the position of the zero and nonzero amplitude subcarriers with the combinatory rule. The paper [6] has proposed such method to reduce the peak-to-average power ratio (PAPR) of OFDM system without reducing the bandwidth efficiency and without increasing the bit error probability. The system was named as parallel combinatory OFDM (PC-OFDM) systems.

On the other hand, in the OFDM systems, each subcarrier transmits data using orthogonal frequency spacing $\Delta f_{0}$. Usually, $\Delta f_{0}$ is chosen as the reciprocal of symbol duration $T_{0}$. Therefore, the normalized symbol duration (or modulation index) of the OFDM system is equal to one $\left(\Delta f_{0} T_{0}=1\right)$. Due to multipath fading and Doppler effect of wireless channel, standard OFDM systems often utilize a guard interval (GI) $T_{g}$ to combat intersymbol interference (ISI), then the normalized symbol duration of the standard OFDM systems can be regarded as $\Delta f_{0}\left(T_{0}+T_{g}\right)>1$. According to BalianLow theorem [7], [8], the OFDM systems must utilize entire duration $\left(\triangle f_{0} T_{0} \geq 1\right)$ to keep orthogonality between subcarriers and to minimize the intercarrier interference (ICI), otherwise, if $\Delta f_{0} T_{0}<1$, the ICI cannot be avoided.

The OFDM system achieves a high transmission rate using high order $M$-QAM constellation. However, with increasing the size $M$ of constellation of $M$-QAM, the implementation complexity of the demodulator increases. To alleviate such an influence, the complicated methods and exorbitant devices must be adopted [9]. In addition, since wireless channel is subject to severe propagation impairment which results in a serious degradation in the link carrier-tonoise ratio (CNR), even the efficient techniques of compensation are used, high order $M$-QAM OFDM cannot achieve the optimal performance. For example, Morinaga et al. have claimed that QPSK is the optimum multilevel modulation for high capacity cellular systems and the option of higher modulation levels will just reduce the system bandwidth efficiency (BWE) [10]. We have designed one novel high compaction multi-carrier modulation (HC-MCM) system which can make the normalized symbol duration smaller than one [11]-[14]. Compared with $T_{0}$ or $\Delta f_{0}$ of OFDM system, HCMCM system can transmit the equal bits of data using $T$ $\left(<T_{0}\right)$ or $\Delta f\left(<\Delta f_{0}\right)$. Only utilizing BPSK or QPSK modulation, the HC-MCM can achieve the transmission rate of high order $M$-QAM OFDM. 
The BWE of HC-MCM can be increased by employing smaller $\Delta f T$, but ICI, which deteriorates bit-error rate (BER) performance, will also be increased with smaller $\Delta f T$. Therefore, we apply the parallel combinatory coding to the HC-MCM system to reduce the ICI since zeroamplitude subcarriers will reduce the ICI if $\Delta f T<1$. On the other hand, by an appropriate choice of the parallel combinatory coding, we can achieve the identical BWE of the HC-MCM system using larger $\Delta f T$.

In this paper, based on the PC-OFDM and HC-MCM, we propose a novel modulation, that is, parallel combinatory/high compaction multi-carrier modulation (PC/HC$\mathrm{MCM}$ ). Two types of PC/HC-MCM systems, which are named as modulated $\mathrm{PC} / \mathrm{HC}-\mathrm{MCM}$ system and (unmodulated) PC/HC-MCM system, can be designed. The modulated PC/HC-MCM system can achieve better BER performance than that of HC-MCM system with the equal BWE by employing appropriate parallel combinatory coding. The PC/HC-MCM system can obtain better peakto-average power ratio (PAPR) characteristics by selecting the suitable constellations for its subcarriers. Furthermore, since the PC/HC-MCM can divide the PC-OFDM symbol duration into multiple time-slots, the advantages of frequency hopping (FH) can be applied in the PC/HC-MCM system. Therefore, we also combine the $\mathrm{PC} / \mathrm{HC}-\mathrm{MCM}$ and frequency hopping multiple access (FHMA) to propose a new multiple access (MA) system. This MA system can synchronously transmit multiple users' data within one symbol duration of PC-OFDM.

The remainder of this paper is organized as follows. The PC-OFDM and PC/HC-MCM models are introduced in Sect. 2. The BWE, BER performance and PAPR of both $\mathrm{PC} / \mathrm{HC}-\mathrm{MCM}$ systems are presented in Sect. 3. We show the performance of multiple access for the downlink system when the system adopts the PC/HC-MCM with frequency hopping in Sect. 4. Conclusions are presented in Sect. 5.

\section{Parallel Combinatory/High Compaction Multi- Carrier Modulation Systems}

\subsection{Parallel Combinatory OFDM Systems}

PC-OFDM [4]-[6] is based on expanding $M$-PSK signal constellation with one extra, zero-amplitude, point. From this larger signal constellation, containing $(M+1)^{N_{C}}\left(N_{C}\right.$ is the number of subcarriers) different waveforms, a subset of waveforms with lower PAPR may be chosen. If chosen properly, compared with the original OFDM system, the system with new signal constellation can realize least bandwidth requirement and lower BER.

The PC-OFDM system can be viewed as two subsystems, that is, original symbol transmission implemented by nonzero modulated subcarriers and combinatory bits of information transmission decided by the position of nonzero and zero subcarriers. At first, the system sets the number of nonzero subcarriers, $N_{P C}$, and the number of zero subcarriers, $N_{C}-N_{P C}$, respectively. Then $N_{P C}$ nonzero subcarriers are modulated by $M$-ary PSK. Here we map $m_{P C}$ [bits] into a subset of subcarriers and $m_{P S K}$ [bits] into the phase of the selected subcarriers. For this case $m_{P S K}$ [bits] can be represented by

$$
m_{P S K}=N_{P C} \log _{2} M .
$$

Selecting $N_{P C}$ subcarriers out of $N_{C}$ subcarriers can be realized in $\left(\begin{array}{c}N_{C} \\ N_{P C}\end{array}\right)$ different ways, so the combinatory bits per PC-OFDM symbol, $m_{P C}[$ bits], can be represented by

$$
m_{P C}=\left\lfloor\log _{2}\left(\begin{array}{c}
N_{C} \\
N_{P C}
\end{array}\right)\right\rfloor,
$$

where $\lfloor x\rfloor$ is the largest integer smaller than or equal to $x$.

Therefore, the number of bits per PC-OFDM symbol $m_{\text {tot }}$ is

$$
m_{\text {tot }}=m_{P S K}+m_{P C}=N_{P C} \log _{2} M+\left\lfloor\log _{2}\left(\begin{array}{c}
N_{C} \\
N_{P C}
\end{array}\right)\right\rfloor .
$$

When $\left(N_{C}-N_{P C}\right) \log _{2} M<\left\lfloor\log _{2}\left(\begin{array}{c}N_{C} \\ N_{P C}\end{array}\right)\right\rfloor$, the PC-OFDM will achieve higher bandwidth efficiency than the OFDM systems with $N_{C}$ subcarriers of $M$-QAM constellation.

By the demodulation, the data that consist of $m_{P S K}$ [bits] can be first recovered and then the $m_{P C}$ bits can be obtained by the combinatory rule with the position of the $N_{C}$ zero and non-zero subcarriers.

\subsection{Parallel Combinatory/High Compaction Multi-Carrier Modulation (PC/HC-MCM)}

\subsubsection{Spectra of PC/HC-MCM Signal}

The PC-OFDM system transmits subcarriers of symbol duration $T_{0}[\mathrm{~s}]$ arranged with a minimum orthogonal frequency spacing $\Delta f_{0}=1 / T_{0}[\mathrm{~Hz}]$ for $M$-PSK and $M$-QAM as shown in Fig. 1(a)). Therefore, the PC-OFDM system can be characterized as a system with a modulation index $\Delta f_{0} T_{0}=$ 1. The modulation index $\Delta f_{0} T_{0}$ can be interpreted as a normalized frequency spacing or normalized symbol duration. Therefore, high compaction multi-carrier modulation (HC-MCM [11], [12]) system can basically be characterized by modulation indices $\Delta f T<1$. We utilize the HC-MCM and PC-OFDM to design the new modulation system called PC/HC-MCM. Figure 1(b) illustrates the spectrum of a $\mathrm{PC} / \mathrm{HC}-\mathrm{MCM}$ signal for a modulation index $\Delta f T=0.375$. In this case, the transmission rate is identical to that of the PC-OFDM illustrated in Fig. 1(a) ( $\left.T=T_{0}, \Delta f=0.375 \Delta f_{0}\right)$, because the bandwidth of each individual subcarrier is identical to that of the PC-OFDM illustrated in Fig. 1(a), whereas the frequency spacing is smaller than that of the PC-OFDM. Thus, the PC/HC-MCM can achieve an identical transmission rate to the PC-OFDM with a narrower bandwidth.

Another form of the spectrum of a PC/HC-MCM signal is illustrated in Fig. 1(c). The modulation index for the spectrum of Fig. 1(c) is identical to that of Fig. 1(b). In this case, 


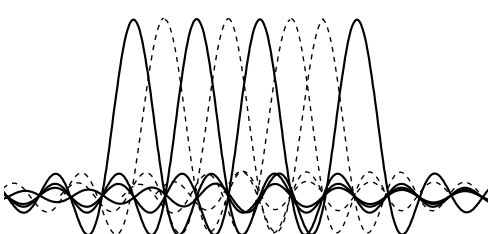

(a) Spectrum of a PC-OFDM signal

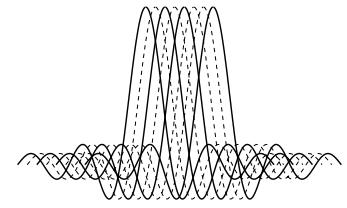

(b) Spectrum of a PC/HC-MCM signal (1)

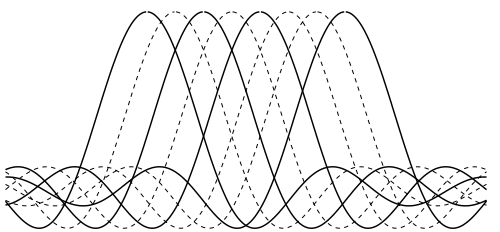

(c) Spectrum of a PC/HC-MCM signal (2)

Fig. 1 Spectra of a PC-OFDM and PC/HC-MCM $(\Delta f T=0.375)$. For $(8,4) \mathrm{PC} / \mathrm{HC}-\mathrm{MCM}$, only 4 subcarriers are transmitted in each symbol duration (SD), e.g. the solid lines for the first SD and dotted lines for the next SD.

the frequency spacing is identical to that the PC-OFDM illustrated in Fig. 1(a) $\left(\Delta f=\Delta f_{0}, T=0.375 T_{0}\right)$. However, a fast transmission rate can be achieved, because the bandwidth of each individual subcarrier is larger than that of the PC-OFDM.

Compared to the PC-OFDM, the PC/HC-MCM can change BWE by adopting different $\Delta f T$. The $\mathrm{PC} / \mathrm{HC}$ MCM can be classified into two systems which are named as the modulated PC/HC-MCM system and the (unmodulated) PC/HC-MCM system. The modulated PC/HC-MCM system adopts $m_{t o t}$ [bits] in (3) for each PC/HC-MCM waveform to transmit data. The PC/HC-MCM system only employs $m_{P C}$ [bits] in (2) for each PC/HC-MCM waveform to transmit data, that is, the transmitter chooses $N_{P C}$ from $N_{C}$ subcarriers to transmit $e^{j \theta_{i}}$ and $\theta_{i}\left(i=1, \ldots, N_{P C}\right)$ can be arbitrary values, but other $\left(N_{C}-N_{P C}\right)$ subcarriers adopt zero-amplitude points to generate different PC/HC-MCM waveforms. Both systems can achieve the higher BWE than that of the $M$-QAM modulated OFDM system with $N_{C}$ subcarriers if the systems choose appropriate combination of $\left(N_{C}, N_{P C}\right)$ and $\Delta f T$.

\subsubsection{Modulated PC/HC-MCM System Model}

Figure 2 shows the transmitter and receiver for the modulated PC/HC-MCM system. Suppose $m_{t o t}$ [bits] (or $m_{P C}$ [bits] for PC/HC-MCM system) can be transmitted in each symbol duration using the specific combination $\left(N_{C}, N_{P C}\right)$ and modulation. It can be expressed with a vector whose elements are $x(k)\left(k=0, \ldots, N_{C}-1\right)$ including $N_{P C}$ modulated subcarriers and $\left(N_{C}-N_{P C}\right)$ zeros. In the transmitter of modulated PC/HC-MCM, $K_{0}$ zeros are tacked on to the

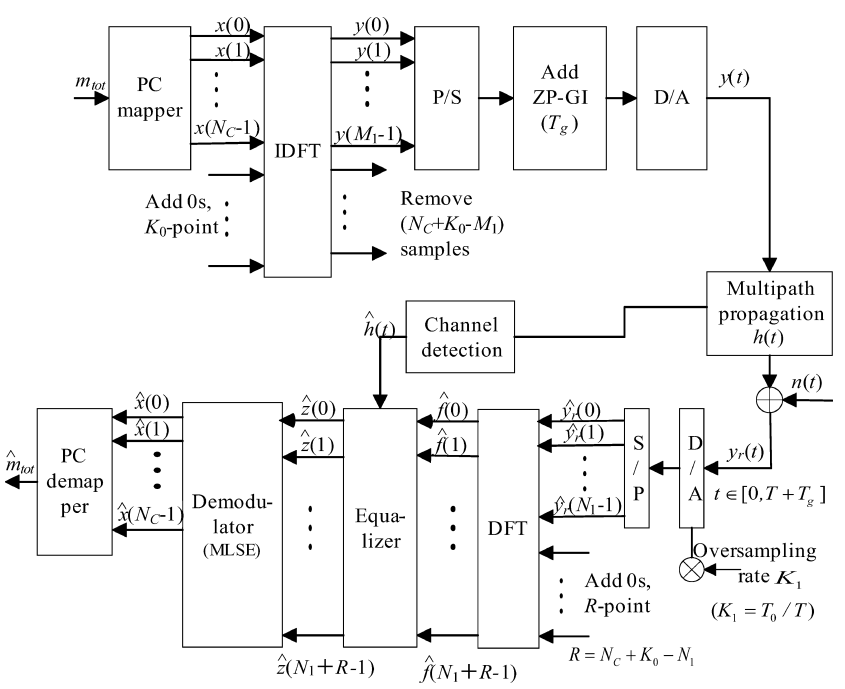

Fig. 2 Transmitter and receiver of the modulated PC/HC-MCM system.

subcarrier symbols $x(k)$ as padding at the input of the IDFT and $\left(N_{C}+K_{0}-M_{1}\right)$ samples are removed at the IDFT output. The operation of padding zeros is for mitigating the aliasing in the frequency domain caused by the IDFT and for interpolation for the samples in the time domain. When the entire samples produced by the inverse discrete Fourier transform (IDFT) are transmitted, the transmitter output $y(t)$ forms an ordinary PC-OFDM waveform. In the modulated PC/HC-MCM transmitter, only a portion of IDFT output samples is transmitted. This operation makes its transmitting symbol compact in the time domain, and makes its spectrum high density in the frequency domain, relative to its transmission rate. Suppose $M_{1}$ samples out of $N_{C}+K_{0}$, i.e., $y(m)\left(m=0, \ldots, M_{1}-1\right)$, are transmitted as shown in Fig. 2 , in this case, the modulation index $\Delta f T$ for modulated $\mathrm{PC} / \mathrm{HC}-\mathrm{MCM}$ signals can be represented as $M_{1} /\left(N_{C}+K_{0}\right)$. After IDFT and parallel-to-serial (P/S) conversion process, several zeros are padded as the postfix guard interval (GI) to alleviate the influence of multipath fading. The duration of zero postfix (ZP) is $T_{g}$. The reason we choose $\mathrm{ZP}$ as GI is that such a method simplifies the demodulation and equalization of the receiver [15]. After digital-to-analog (D/A) conversion, the modulated PC/HC-MCM signal $y(t)$ is transmitted into the channel.

For simplicity, the time-domain modulated PC/HCMCM signal is expressed in complex base-band notation as

$$
y(t)=\sum_{n=0}^{N_{C}-1} x(n) e^{j 2 \pi n \Delta f t}, \quad t \in[0, T]
$$

Suppose that the impulse response $h(t)$ of the fading channel is

$$
h(t)=\sum_{i=1}^{L_{P}} A_{i} e^{j \theta_{i}} \delta\left(t-\tau_{i}\right) \quad t \in\left[0, T+T_{g}\right],
$$

where $L_{P}$ means the number of paths and each path has the relative path delay $\tau_{i}\left(\tau_{1} \leq \tau_{2} \leq \ldots \leq \tau_{L_{P}}<T_{g}\right) . A_{i}=A_{i}(t)$ 
and $\theta_{i}=\theta_{i}(t)$ are the slowly Rayleigh-fading amplitude attenuation and phase rotation of each path. We assume $A_{i}$ and $\theta_{i}$ are constant during each $\mathrm{PC} / \mathrm{HC}-\mathrm{MCM}$ symbol duration.

Therefore the received signal, $y_{r}(t)\left(t \in\left[0, T+T_{g}\right]\right)$, can be represented as

$$
\begin{aligned}
y_{r}(t) & =h(t) \otimes y(t)+n(t) \\
& =\sum_{i=1}^{L_{P}} A_{i} e^{j \theta_{i}} y\left(t-\tau_{i}\right)+n(t) \quad t \in\left[0, T+T_{g}\right],
\end{aligned}
$$

where $n(t)$ is the additive white Gaussian noise (AWGN).

The receiver generates $N_{1}$ discrete-time samples, $\hat{y}_{r}(m)\left(m=0, \ldots, N_{1}-1\right)$, from the received signal $y_{r}(t)$ after analog-to-digital (A/D) conversion with oversampling rate $K_{1}$. We assume $K_{1}=T_{0} / T$ and the number of time-domain samples of GI is $N_{g}$. The $N_{1}$ received samples, $\hat{y}_{r}(m)$, followed by $R$ zeros yield $\hat{f}(n)\left(n=0, \ldots, N_{1}+R-1\right)$ by DFT, as shown in Fig. 2. Here $R\left(R=K_{1} N-N_{1}\right)$ is the number of samples corresponding to the vanished period of the entire PC-OFDM samples. If $T+T_{g}>T_{0}$, we assume that $R=0$ and the samples from $y_{r}(t)$ during $\left[T_{0}, T+T_{g}\right]$ will be overlapped with the samples of $y_{r}(t)$ during $\left[0, T+T_{g}-T_{0}\right]$, which is identical to the process that ZP-OFDM utilizes the guard interval to eliminate the ISI [15]. In such a case, $N_{1}$ will be decreased to $N_{1} T_{0} /\left(T+T_{g}\right)$. It should be pointed out that we can set arbitrary integers for $N_{1}$ and $R$ such that the relations $K_{1}=T_{0} / T=\left(R+N_{1}\right) /\left(N_{1}-N_{g}\right)=\left(N_{C}+K_{0}\right) / M_{1}$ and $N_{C} \leq N_{1}-N_{g}$ hold. For simplicity of analysis, we assume that $R+N_{1}=N_{C}+K_{0}$ and $M_{1}=N_{1}-N_{g}$.

Then the DFT outputs $\hat{f}(n)\left(n=0, \ldots, N_{1}+R-1\right)$ are equalized with the estimation $\hat{h}(t)$ of the channel impulse response $h(t)$. The goal of equalization is to reduce the multipath interference and we adopt the zero-forcing equalizer as described below.

Suppose each path delay $\tau_{i}$ corresponds to the duration of $N_{\tau_{i}}\left(i=1, \ldots, L_{P}\right)$ samples after A/D conversion. At the receiver front, discrete-time samples $\hat{y}_{r}(m)\left(m=0, \ldots, N_{1}-\right.$ 1) of the received signal $y_{r}(t)$ are

$$
\begin{aligned}
\hat{y}_{r}(m)= & \sum_{i=1}^{L_{P}} A_{i} e^{j \theta_{i}} \sum_{k=0}^{N_{C}-1} x(k) e^{j \frac{2 \pi k\left(m-N_{\tau_{i}}\right)}{N_{1}+R}} \\
& \times\left[u\left(m-N_{\tau_{i}}\right)-u\left(m-N_{\tau_{i}}-M_{1}+1\right)\right]+n(m),
\end{aligned}
$$

where $n(m)$ is the discrete value of $n(t)$ and $u(m)$ is the unit step function. Therefore, after DFT operation, as was shown in Fig. 2, the receiver output $\hat{f}(n)\left(n=0, \ldots, N_{1}+R-1\right)$ can be expressed as

$$
\begin{aligned}
\hat{f}(n)= & \frac{1}{N_{1}+R}\left(\sum_{i=1}^{L_{P}} A_{i} e^{j \theta_{i}} \sum_{k=0}^{N_{C}-1} x(k) e^{-j \frac{2 \pi n N_{\tau_{i}}}{N_{1}+R}}\right. \\
& \left.\times \sum_{m=0}^{M_{1}-1} e^{j \frac{2 \pi m(k-n)}{N_{1}+R}}+n^{\prime}(n)\right)
\end{aligned}
$$

where $n^{\prime}(n)$ is the frequency response of $n(m)$.

Suppose that $H(f)$ is the frequency response of channel $h(t)$ and its discrete representation is $H(n)\left(n=0, \ldots, N_{1}+\right.$ $R-1)$, where $H(n)=\left.H(f)\right|_{f=n f_{0}}$. Then the weight that the zero-forcing equalizer will process in order to compensate the channel impairment can be defined as

$$
H_{Z F}(n)=H^{-1}(n)=\left(\frac{1}{N_{1}+R} \sum_{i=1}^{L_{P}} A_{i} e^{j \theta_{i}} e^{-j \frac{2 \pi n N_{\tau_{i}}}{N_{1}+R}}\right)^{-1} .
$$

After being passed through the equalizer, the received signal $\hat{f}(n)$ will be changed to $\hat{z}(n)$. $\hat{z}(n)\left(n=0, \ldots, N_{1}+R-1\right)$ can be expressed as

$$
\begin{aligned}
\hat{z}(n) & =\hat{f}(n) \times H_{Z F}(n) \\
= & \frac{\sum_{i=1}^{L_{P}} A_{i} e^{j \theta_{i}} \sum_{k=0}^{N_{C}-1} e^{-j \frac{2 \pi n N_{\tau_{i}}}{N_{1}+R}} x(k) \sum_{m=0}^{M_{1}-1} e^{j \frac{2 \pi m(k-n)}{N_{1}+R}}+n^{\prime}(n)}{\sum_{i=1}^{L_{P}} A_{i} e^{j \theta_{i}} e^{-j \frac{2 \pi n N_{\tau_{i}}}{N_{1}+R}}} .
\end{aligned}
$$

Therefore, the perfect zero-forcing equalizer can remove the multipath interference, and $\hat{z}(n)$ can be expressed as

$$
\begin{aligned}
\hat{z}(n) & =\sum_{m=0}^{M_{1}-1} \sum_{k=0}^{N_{C}-1} x(k) e^{j \frac{2 \pi m(k-n)}{N_{1}+R}}+n^{\prime \prime}(n) \\
& =\sum_{k=0}^{N_{C}-1} x(k) \frac{1-e^{j \frac{2 \pi M_{1}(k-n)}{N_{C}+K_{0}}}}{1-e^{j \frac{2 \pi(k-n)}{N_{C}+K_{0}}}}+n^{\prime \prime}(n) \\
& =\sum_{k=0}^{N_{C}-1} x(k) \frac{1-e^{j 2 \pi(k-n) \frac{T}{T_{0}}}}{1-e^{j \frac{2 \pi(k-n)}{M_{1}} \frac{T}{T_{0}}}}+n^{\prime \prime}(n),
\end{aligned}
$$

where $n^{\prime \prime}(n)=n^{\prime}(n) /\left(\sum_{i=1}^{L_{P}} A_{i} e^{j \theta_{i}} e^{-j \frac{2 \pi n N_{\tau_{i}}}{N_{1}+R}}\right)$.

Finally, the estimates $\hat{x}(k)\left(k=0, \ldots, N_{C}-1\right)$ of $x(k)$ that contains $m_{\text {tot }}$ [bits] are recovered through the demodulation stage with the samples $\hat{z}(n)\left(n=0, \ldots, N_{1}+R-1\right)$.

From (9), we can find that the ICI will appear if $\Delta f T<1$. The modulated $\mathrm{PC} / \mathrm{HC}-\mathrm{MCM}$ cannot keep orthogonality between subcarriers. Therefore, the usual demodulation algorithm, that is, each subcarrier is demodulated independently, cannot be utilized in the modulated PC/HC-MCM systems. A reasonable approach for attaining a good demodulation performance will be the maximum likelihood sequence estimation (MLSE) at the decision stage in the receiver. The BER performance of the MLSE often depends on the minimum Euclidean distance (MED) between all modulated PC/HC-MCM waveforms. The computation process of the MED can be shown in the following example. Since BPSK-modulated PC/HCMCM with $\left(N_{C}, N_{P C}\right)=(8,4)$ can generate 1120 different vectors $\mathbf{X}_{i}(i=1, \ldots, 1120)$ whose elements are $x_{i}(k)(k=$ $\left.0, \ldots, N_{C}-1\right)$, each vector will generate the samples $z_{i}(n)$ $\left(z_{i}(n)=\hat{z}_{i}(n)-n_{i}^{\prime \prime}(n) ; n=0, \ldots, N_{1}+R-1\right)$ of (9) after the equalization of receiver without the noise. Therefore, we can calculate a value of the $\operatorname{MED} D_{i}(i=1, \ldots, 1120)$ for $\mathbf{X}_{i}$ using other vectors $\mathbf{X}_{j}(j=1, \ldots, 1120 ; j \neq i)$ as follows:

$$
D_{i}=\min _{j}\left(\sqrt{\sum_{n=0}^{N_{1}+R-1}\left|z_{i}(n)-z_{j}(n)\right|^{2}}\right) \text {. }
$$


We evaluate the minimum value $\min _{i}\left(D_{i}\right)(i=1, \ldots, 1120)$ of $D_{i}$ as the MED of modulated PC/HC-MCM. We also evaluate the average MED (AMED) $\left(\sum_{i=1}^{1120} D_{i} / 1120\right)$ to represent an average property.

Figure 3 shows the MED and AMED properties as a function of $\Delta f T$ for two modulations: A is the BPSKmodulated PC/HC-MCM with $\left(\left(N_{C}, N_{P C}\right)=(8,4)\right)$ and $\mathrm{B}$ is the PC/HC-MCM with $\left(\left(N_{C}, N_{P C}\right)=(8,4)\right)$. Signal power per bit is assumed to be unity. For a specific value of $\Delta f T$, the transmission rate of modulated $\mathrm{PC} / \mathrm{HC}$ MCM becomes faster than that of the PC-OFDM by a factor of $1 /(\Delta f T)$, provided that the frequency spacing $\Delta f$ of the modulated PC/HC-MCM is identical to that of the PCOFDM $\Delta f_{0}$ (e.g., the transmission rate of BPSK-modulated $\mathrm{PC} / \mathrm{HC}-\mathrm{MCM}$ with $\Delta f T=0.125$ is identical to that of 1024 QAM-modulated OFDM). The AMED for the modulated PC/HC-MCM becomes larger than that of the OFDM when the transmission rate are identical, as can been seen in Fig. 3 . Therefore, only using BPSK and QPSK modulation, modulated PC/HC-MCM can achieve the transmission rate of high $M$-QAM OFDM. Figure 3 also shows that the smaller $\Delta f T$ will decrease the MEDs and AMEDs that correponds to the larger ICI.

Since the amount of computations required for Euclidean distance increases exponentially with $N_{C}$, the MLSE can only be utilized for small $N_{C}$. For large $N_{C}$, a demodulation method using the $M$-algorithm, which the calculation complexity preserves a linear increase with $N_{C}$, has been proposed [14]. In this study, we adopt the MLSE to obtain $\hat{x}(i)\left(i=1, \ldots, N_{c}-1\right)$. After that, utilizing the rule of PC mapper, the system can recover the $m_{t o t}$ [bits] of data.

On the other hand, the spectrum illustrated in Fig. 1(b) can be obtained by time-scaling the duration of the waveform, whose spectrum is illustrated in Fig. 1(c), to the equal time duration of the original PC-OFDM waveform. The time-scaling can be accomplished by an appropriate choice of D-A conversion rate, or by adjusting the zero-padding in the transmitter.

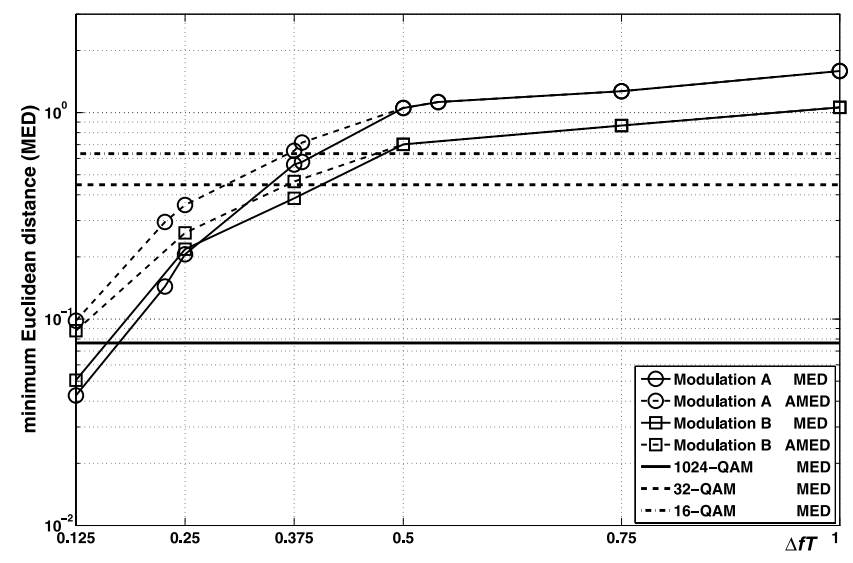

Fig. 3 The minimum Euclidean distances (MEDs) and average MEDs (AMEDs) of two modulations with different $\Delta f T$. Modulation A: BPSKmodulated PC/HC-MCM with $\left(N_{C}, N_{P C}\right)=(8,4)$; Modulation B: PC/HC$\mathrm{MCM}$ with $\left(N_{C}, N_{P C}\right)=(8,4)$ and $e^{j \theta_{i}}=1$.

\section{Performance of PC/HC-MCM System}

\subsection{Bandwidth Efficiency of PC/HC-MCM}

The BWE of multicarrier systems depends on the number of subcarriers and the type of pulse shaping employed. For ordinary OFDM system with $M$-QAM, the BWE $\eta_{1}$ will be

$$
\eta_{1}=\frac{\left(N_{C} \log _{2} M\right) / T_{0}}{2 / T_{0}+\left(N_{C}-1\right) \Delta f_{0}}=\frac{N_{C} \log _{2} M}{N_{C}+1} .
$$

And for HC-MCM system with $M$-QAM, the BWE $\eta_{2}$ will be

$$
\eta_{2}=\frac{\left(N_{C} \log _{2} M\right) / T}{2 / T+\left(N_{C}-1\right) \Delta f}=\frac{N_{C} \log _{2} M}{2+\left(N_{C}-1\right) \Delta f T} .
$$

We define the BWE $\eta_{3}$ of the modulated PC/HC-MCM system and $\eta_{4}$ of the PC/HC-MCM system as follows:

$$
\begin{aligned}
& \eta_{3}=\frac{m_{t o t} / T}{2 / T+\left(N_{C}-1\right) \Delta f}=\frac{N_{P C} \log _{2} M+\left\lfloor\log _{2}\left(\begin{array}{c}
N_{C} \\
N_{P C}
\end{array}\right)\right\rfloor}{2+\left(N_{C}-1\right) \Delta f T}, \\
& \eta_{4}=\frac{m_{P C} / T}{2 / T+\left(N_{C}-1\right) \Delta f}=\frac{\left\lfloor\log _{2}\left(\begin{array}{c}
N_{C} \\
N_{P C}
\end{array}\right)\right\rfloor}{2+\left(N_{C}-1\right) \Delta f T} .
\end{aligned}
$$

Figure 4 plots the BWEs of above four systems. We choose $N_{C}=8$ for BPSK-modulated HC-MCM and $N_{C}=$ 32 for QPSK-modulated HC-MCM systems, respectively. We choose for the BPSK- and QPSK-modulated PC/HCMCMs, $\left(N_{C}, N_{P C}\right)=(8,4)$ and $(32,28)$, respectively, that maximize the value of $m_{\text {tot }}$. For PC/HC-MCM system, $\left(N_{C}, N_{P C}\right)=(8,4)$ and $(32,16)$ are chosen to maximize the values of $m_{P C}$. From (12), (13) and Fig. 3, the modulated PC/HC-MCM system will improve the BWE of the HC-MCM system if $\left(N_{C}-N_{P C}\right) \log _{2} M<\left\lfloor\log _{2}\left(\begin{array}{c}N_{C} \\ N_{P C}\end{array}\right)\right\rfloor$ holds. Since the ICI will be enlarged by smaller values of $\Delta f T$, we can employ larger $\Delta f T$ of the modulated $\mathrm{PC} / \mathrm{HC}-\mathrm{MCM}$ system to achieve identical BWE of HC-MCM system using

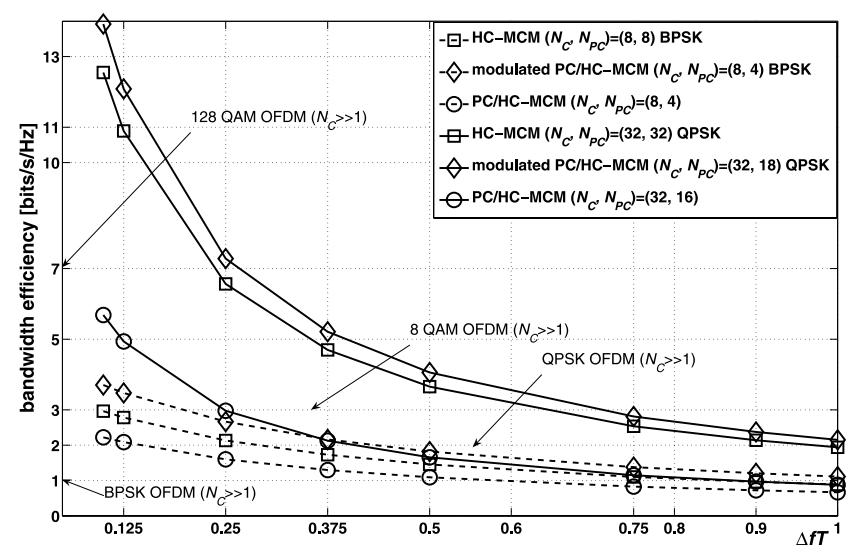

Fig. 4 Bandwidth efficiency for four systems. 
the appropriate combination $\left(N_{C}, N_{P C}\right)$. For example, the BWEs of the BPSK-modulated HC-MCM systems $\left(N_{C}=8\right)$ with $\Delta f T=0.125,0.25$ and 0.375 will be identical to that of the BPSK-modulated PC/HC-MCM systems $\left(\left(N_{C}, N_{P C}\right)\right.$ $=(8,4))$ with $\Delta f T=0.227,0.384$ and 0.54 , respectively. And the BWEs of the QPSK-modulated HC-MCM systems $\left(N_{C}=32\right)$ with $\Delta f T=0.125,0.25$ and 0.375 will be identical to that of the QPSK-modulated PC/HC-MCM systems $\left(N_{C}, N_{P C}\right)=(32,28)$ with $\Delta f T=0.145,0.2844$ and 0.4231 , respectively. Therefore, compared to the HC-MCM system, the modulated PC/HC-MCM can decrease the ICI by employing the larger $\Delta f T$.

For the PC/HC-MCM system, we can still achieve the identical BWE to the $M$-QAM OFDM using an appropriate combination of $\left(N_{C}, N_{P C}\right)$ and $\Delta f T$. However, the modulated PC/HC-MCM system will outperform the PC/HCMCM system because of $m_{P C}<m_{t o t}$ for any specific $\left(N_{C}, N_{P C}\right)$. Whereas the PC/HC-MCM system can choose arbitrary value of $e^{j \theta}\left(i=1, \ldots, N_{P C}\right)$ for $N_{P C}$ subcarriers. Therefore the PC/HC-MCM system can reduce the PAPR.

\subsection{Simulated BER Performance of Modulated PC/HC MCM System over AWGN Channel}

Common specifications of simulations are listed in Table 1. Figure 5 shows BER performance of BPSK-modulated PC/HC-MCM system with $\left(N_{C}=8, N_{P C}=4\right)$ over the AWGN channel. The modulation indices $\Delta f T$ are 0.227 , 0.384 and 0.54, which have the identical BWEs to the BPSK-modulated HC-MCM system with $N_{C}=8$ and $\Delta f T=0.125,0.25$ and 0.375 , respectively. We also plot the theoretical results for the $M$-QAM-modulated OFDM with $N_{C}=8$ and $M=16$ and 256, since their transmission rates are equal to the modulated $\mathrm{PC} / \mathrm{HC}-\mathrm{MCM}$ system with $\Delta f T=0.384$ and 0.227 , respectively, provided that the frequency spacing of $\mathrm{PC} / \mathrm{HC}-\mathrm{MCM}(\Delta f)$ is identical to that of the OFDM system $\left(\Delta f_{0}\right)$. From the results, we can find that the BPSK-modulated PC/HC-MCM system can achieve better BER performance. For $\Delta f T=0.227,0.384$ and $\mathrm{BER}=$ $10^{-4}$, the modulated PC/HC-MCM system obtains $7.4[\mathrm{~dB}]$ gain and $1.25[\mathrm{~dB}]$ gain compared to the 256-QAM OFDM and 16-QAM OFDM systems, respectively.

It should be pointed out that the BWEs of 16-QAM and 256-QAM OFDM are higher than that of the BPSKmodulated HC-MCM system with $\left(N_{C}=8\right)$ and $\Delta f T=$ $0.25,0.125$ because both edges of HC-MCM spectra shown in Fig. 1(c) are increased with smaller $\Delta f T$. Such a loss of BWE can be alleviated by increasing $N_{C}$. From Fig. 3, we can find that the BWEs of QPSK and 8-QAM OFDM are

Table 1 Specifications of simulations.

\begin{tabular}{l|l}
\hline \hline System Item & Parameter \\
\hline \hline Combination $\left(N_{C}, N_{P C}\right)$ & $(8,4)$ \\
\hline Subcarrier modulation & BPSK \\
\hline Synchronization & Complete \\
\hline Demodulation & MLSE \\
\hline Channel type & AWGN \\
\hline
\end{tabular}

identical to that of the above BPSK-modulated HC-MCM systems. The BER performance of QPSK and 8-QAM OFDM is better than that of the BPSK-modulated HC-MCM systems which can be found in Fig. 4. For $\Delta f T=0.125$ and BER $=10^{-4}$, the BPSK-modulated HC-MCM system has $8[\mathrm{~dB}]$ gap between the 8-QAM OFDM system. But the BPSK-modulated PC/HC-MCM system with $\Delta f T=0.227$ will improve $4.5[\mathrm{~dB}]$ for $\mathrm{BER}=10^{-4}$ than that of the BPSK-modulated HC-MCM system.

\subsection{Simulated BER Performance of Modulated PC/HC-} MCM System over Multipath Fading Channel

Specifications of simulations are listed in Table 2. Figure 6 shows BER performance of BPSK-modulated PC/HCMCM system with $\left(N_{C}, N_{P C}\right)=(8,4)$ over a multipath fading channel in comparison with the HC-MCM. We assume the transmission rates of both systems are identical

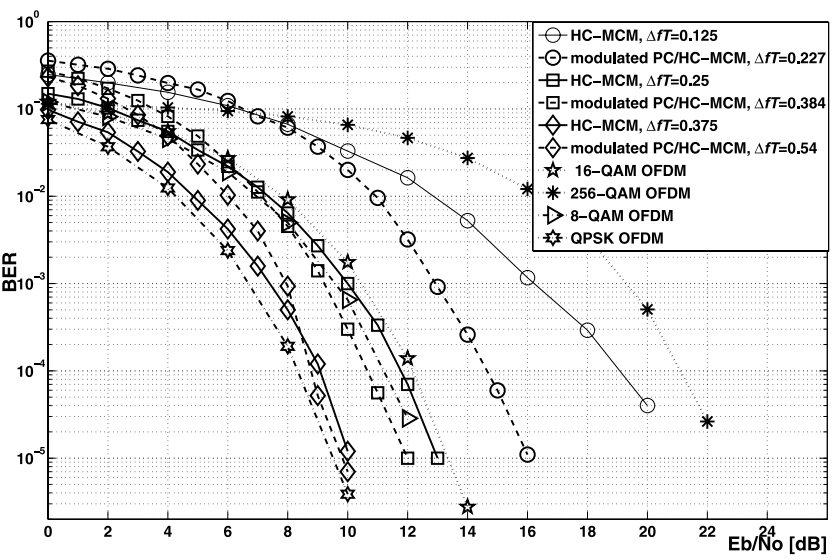

Fig. 5 BER performance of BPSK-modulated PC/HC-MCM system $\left(N_{C}, N_{P C}\right)=(8,4)$ over AWGN channel.

Table 2 Specifications of simulations.

\begin{tabular}{l|l}
\hline \hline System Item & Parameter \\
\hline \hline Subcarrier modulation & BPSK \\
\hline Combination $\left(N_{C}, N_{P C}\right)$ & $\begin{array}{l}(8,4) \text { for PC/HC-OFDM } \\
N_{C}=8 \text { for HC-MCM }\end{array}$ \\
\hline Synchronization & Complete \\
\hline Channel type & JTC' 94 (indoor residential B) \\
& $\left(L_{P}=8\right)$ \\
\hline Equalization & zero-forcing equalization \\
\hline Supported PC/HC-MCM & $570 \mathrm{k}\left(T+T_{g}=1.75 \mu \mathrm{s}\right)$ \\
rate (symbol/second) & \\
\hline Relative delay for & $0,50,100,150$ \\
each path (ns) & $200,250,300,350$ \\
\hline $\begin{array}{l}\text { Relative power attenuation } \\
\text { for each path (dB) }\end{array}$ & $0,-2.9,-5.8,-8.7$, \\
\hline $\begin{array}{l}\text { Maximum Doppler } \\
\text { frequency shift } f_{D}(\mathrm{~Hz})\end{array}$ & $-11.6,-14.5,-17.4,-20.3$ \\
\hline $\begin{array}{l}\text { Duration of zero } \\
\text { guard signal }\end{array}$ & $T_{g}=0.25 T$ \\
\hline$\Delta f T$ & $0.125,0.25,0.375$ for HC-MCM \\
& $0.227,0.384,0.54$ for modulated \\
\hline Noise & Additive White Gaussian Noise \\
\hline
\end{tabular}




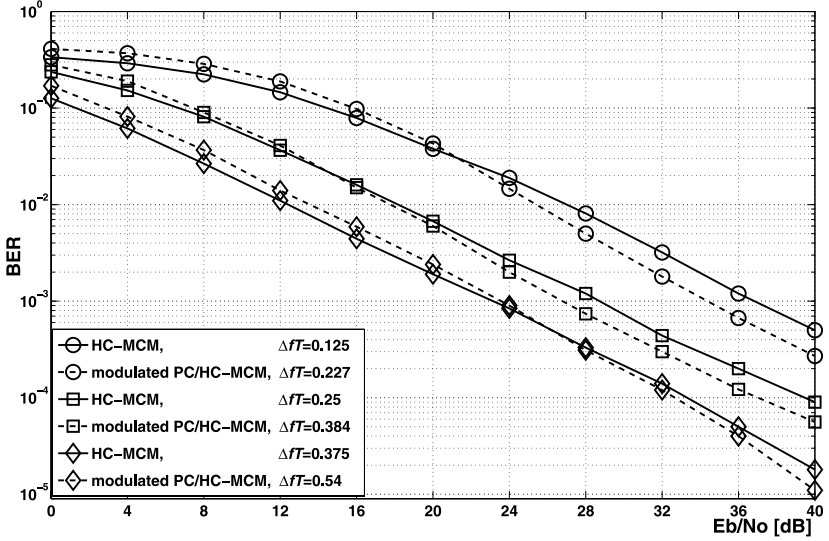

Fig. 6 BER performance of BPSK-modulated PC/HC-MCM system with $\left(N_{C}, N_{P C}\right)=(8,4)$ over a multipath channel.

for all values of $\Delta f T$; thus the frequency spacing $(\Delta f)$ is reduced by decreasing $\Delta f T$. The modulation indices $\Delta f T$ are $0.227,0.384$ and 0.54 , which have the identical BWEs to the BPSK-modulated HC-MCM system $\left(N_{C}=8\right)$ with $\Delta f T=0.125,0.25$ and 0.375 , respectively. We choose the fading channel model of JTC'94 (indoor residential B) [16] to compare the BER performance of both systems. It is assumed that the receiver knows the channel information $h(t)$ and carries out the perfect zero-forcing equalization. From the results, we can find that the BPSK-modulated PC/HCMCM system can achieve better BER performance. For example, using $\Delta f T=0.227$, the modulated PC/HC-MCM system can achieve $3[\mathrm{~dB}]$ gain for $\mathrm{BER}=10^{-3}$ than that of the HC-MCM system with the equal BWE.

\subsection{Simulated BER Performance of PC/HC-MCM System over AWGN Channel}

Specifications are listed in Table 3. Figure 7 shows the characteristics of the bandwidth efficiency (BWE) versus $\mathrm{Eb} / \mathrm{No}$ required for BER $=10^{-4}$. We adopt the combinations of $\left(N_{C}, N_{P C}\right)=(16,5),(16,3),(16,13)$ and $(8,4) . N_{P C}$ subcarriers are modulated by $e^{j \theta_{i}}\left(i=1, \ldots, N_{P C}\right)$ where $\theta_{i}$ is the phase angle of the $i$-th subcarrier. In order to evaluate the impact of the ICI, $\theta_{i}\left(i=1, \ldots, N_{P C}\right)$ are chosen to be random values $(\theta \in[0,2 \pi])$. When $\Delta f T=1$, the PC/HC-MCM system reduces to the PC-OFDM system without $m_{P S K}$. For a specific $\mathrm{Eb} / \mathrm{No}$, different combination and $\Delta f T$ can cause different bandwidth efficiency. For example, when $\mathrm{Eb} / \mathrm{No} \simeq 12.5[\mathrm{~dB}]$, the PC/HC-MCM with $\Delta f T=0.375$ and $\left(N_{C}, N_{P C}\right)=(16,5)$ achieves $\mathrm{BWE} \simeq 1.6[\mathrm{bits} / \mathrm{s} / \mathrm{Hz}]$, whereas the (nomodulated) PC-OFDM with $\Delta f T=1.0$ and $\left(N_{C}, N_{P C}\right)=(8,4)$ achieves $\mathrm{BWE} \simeq 0.7[\mathrm{bits} / \mathrm{s} / \mathrm{Hz}]$. This means that twice the bandwidth efficiency is obtained by the PC/HC-MCM system. On the other hand, the PC/HC-MCM system with combinations of $\left(N_{C}, N_{P C}\right)=(16,3)$ and $(16$, 13) can achieve the equal bandwidth efficiency because the values of $m_{P C}$ for both systems are the same. However, we can find that $\left(N_{C}, N_{P C}\right)=(16,13)$ will consume more power than that of $\left(N_{C}, N_{P C}\right)=(16,3)$.
Table 3 Specifications of simulations.

\begin{tabular}{l|l}
\hline \hline System Item & Parameter \\
\hline \hline Combination $\left(N_{C}, N_{P C}\right)$ & $(8,4),(16,3),(16,13),(16,5)$ \\
\hline \multirow{3}{*}{ Subcarrier modulation } & $\begin{array}{l}N_{P C} \text { subcarriers use } e^{i \theta_{i}}\left(\theta_{i} \text { is }\right. \\
\text { random for } i \text { th subcarrier }), \\
\left(N_{C}-N_{P C}\right) \text { subcarriers } \\
\text { adopt zero-amplitude points }\end{array}$ \\
\hline Synchronization & Complete \\
\hline Demodulation & MLSE \\
\hline Channel type & AWGN \\
\hline
\end{tabular}

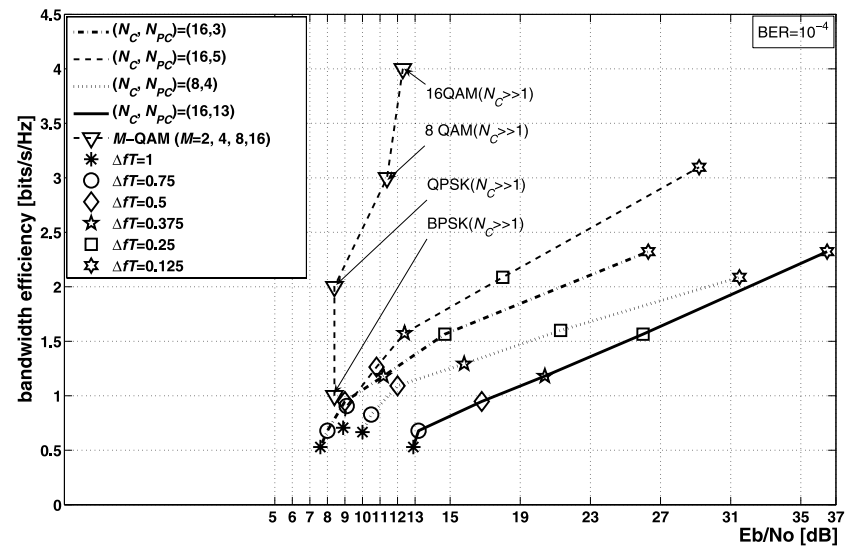

Fig. 7 BER versus Eb/No $[\mathrm{dB}]$ required for the $\mathrm{BER}=10^{-4}$ for $\mathrm{PC} / \mathrm{HC}$ MCM system over AWGN channel.

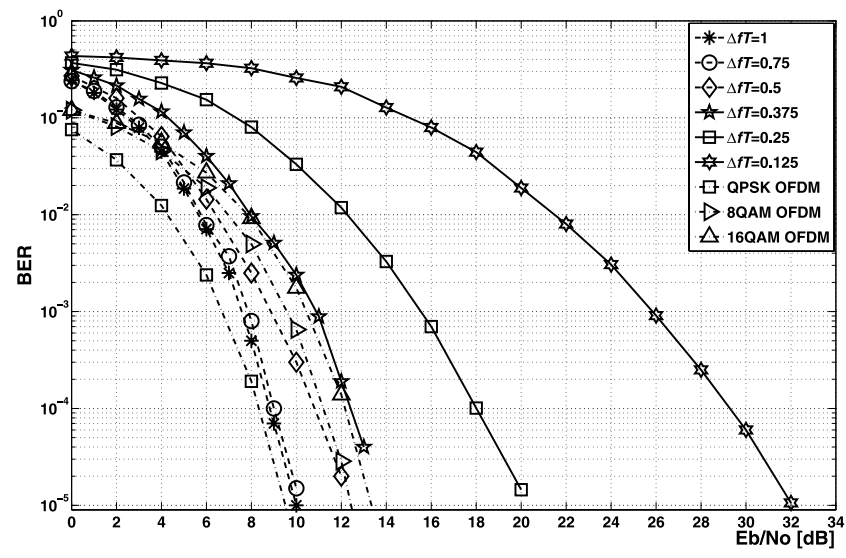

Fig. 8 BER performance of $\mathrm{PC} / \mathrm{HC}-\mathrm{MCM}$ system with combination with $\left(N_{C}, N_{P C}\right)=(16,5)$ over AWGN channel.

Figure 8 shows the simulated BER performance of the PC/HC-MCM system with $\left(N_{C}, N_{P C}\right)=(16,5)$ with the $N_{P C}$ subcarriers and $\theta_{i}\left(i=1, \ldots, N_{P C}\right)$ are chosen to be random values $(\theta \in[0,2 \pi])$. From the results we can find that for the same BER, more power is consumed with the smaller $\Delta f T$ but the BWE can be improved. It is remarkable that the $\mathrm{PC} / \mathrm{HC}-\mathrm{MCM}$ with $\Delta f T=0.5,0.75$ and 1 achieves almost the same BER performance. Note that $\Delta f T=0.375$ also indicates comparable BER performance.

Figure 8 also shows the tradeoff between the BWE and the demodulation complexity of the PC/HC-MCM system. Large number $N_{C}$ of PC/HC-MCM system results in 
high complexity, especially for the MLSE of demodulation. By utilizing the method we proposed to the decision stage [14], it can preserve a linear increase with $N_{C}$ for the calculation complexity. On the other hand, Figs. 7 and 8 also show the BER performance of OFDM system using QPSK, 8 QAM, 16 QAM modulation. PC/HC-MCM system cannot achieve better performance than that of the OFDM systems, but PC/HC-MCM system can realize better characteristics of PAPR which will be presented in following subsection.

\subsection{PAPR of Two PC/HC-MCM Systems}

One of the major drawbacks of OFDM system is that the time-domain OFDM signals exhibit large value of PAPR. Therefore, complicated methods and exorbitant devices must be utilized to OFDM system to decrease the value of PAPR. The PAPR will be enlarged if $N_{C}$ or $M$-QAM modulation order $M$ increases [17].

Let us denote the data block of length $N_{C}$ as a vector $\mathbf{X}=\left[x(0), \ldots, x\left(N_{C}-1\right)\right]$. The symbol duration of the PC/HC-MCM is $T$. Each subcarrier modulates one of the set of frequencies $f_{n}\left(n=0, \ldots, N_{C}-1\right)$ and $f_{n}=n \Delta f$. The complex envelope of the transmitted PC/HC-MCM signal is given by

$$
y(t)=\frac{1}{\sqrt{N_{C}}} \sum_{n=0}^{N_{C}-1} x(n) e^{j 2 \pi n \Delta f t} \quad t \in[0, T] .
$$

The PAPR of the signal of PC/HC-MCM can be defined as

$$
P A P R=\frac{\max _{0<t<T}|y(t)|^{2}}{P_{A V}},
$$

where $P_{A V}$ is the ensemble average of the power of timedomain PC/HC-MCM signals $y(t)$. We randomly generate millions of time-domain PC/HC-MCM signals by computer to obtain the approximate value $\hat{P}_{A V}$ of $P_{A V}$. The simulated $\hat{P}_{A V}$ for modulated PC/HC-MCM signals $\left(N_{C}, N_{P C}\right)=$ $(64,48)$ are almost identical for all values of $\Delta f T$. To capture the peaks of the PC/HC-MCM signal, we adopt 4 times oversampling time-domain samples which can totally approximate the continus time-domain signal [18]. We use the complementary cumulative density function (CCDF) of the PAPR (Prob $\left[\mathrm{PAPR}>\mathrm{PAPR}_{0}\right]$ ) to represent the PAPR characteristics. In the following simulations, we can confirm that the PAPR of modulated PC/HC-MCM system will be reduced by decreasing $\Delta f T$.

For PC-OFDM signals with large $N_{C}$, the peak values of the signals appear uniformly in the PC-OFDM symbol duration $\left[0, T_{0}\right]$. The modulated PC/HC-MCM only utilizes the partial time-domain signal during $[0, T]$ of the entire PC-OFDM time-domain signal $\left(\left[0, T_{0}\right]\right)$ to transmit the equal bits of data, as can be seen in Fig. 2. Therefore, if the peak values of the PC-OFDM signal appears in the duration $\left(T, T_{0}\right]$, the peak values of the modulated PC/HC-MCM signal will be smaller than that of PC-OFDM signal. On the other hand, the simulated $\hat{P}_{A V}$ for the modulated PC/HCMCM signals $\left(N_{C}, N_{P C}\right)=(64,48)$ are almost identical for all values of $\Delta f T$. So it can be intuitively inferred that the PAPR (Prob[PAPR $>$ PAPR $\left._{0}\right]$ ) will be reduced by decreasing $\Delta f T$ compared to the ordinary PC-OFDM waveforms.

Figure 9 gives the CCDFs of the PAPR of the modulated $\mathrm{PC} / \mathrm{HC}-\mathrm{MCM}$ signals. The PC-OFDM is the special case of the modulated PC/HC-MCM when $\Delta f T=1$. QPSK is used to modulate the subcarriers. We use $N_{C}=64$ to simulate the PAPR because large subcarriers can further distinctly exhibit the CCDF property of PAPR during the adjustment of $\Delta f T$. From the results, we can confirm that the PAPR of the modulated PC/HC-MCM system can be reduced with $\Delta f T$.

For the PC/HC-MCM system, we can utilize the values of $e^{j \theta_{i}}\left(i=1, \ldots, N_{P C}\right)$ to obtain small PAPR. In this paper, we assume that a certain set of $\theta_{i}$ is used for all the PC/HC-MCM signals, and that each element of the set takes a value $\theta_{i} \in\{\pi / 2, \pi, 3 \pi / 2,2 \pi\}$. The best set that minimizes the PAPR was searched through the simulations from all $4^{N_{P C}}$ kinds. The results plotted in Fig. 10 indicate that the PAPR is dramatically improved by such a simple way.

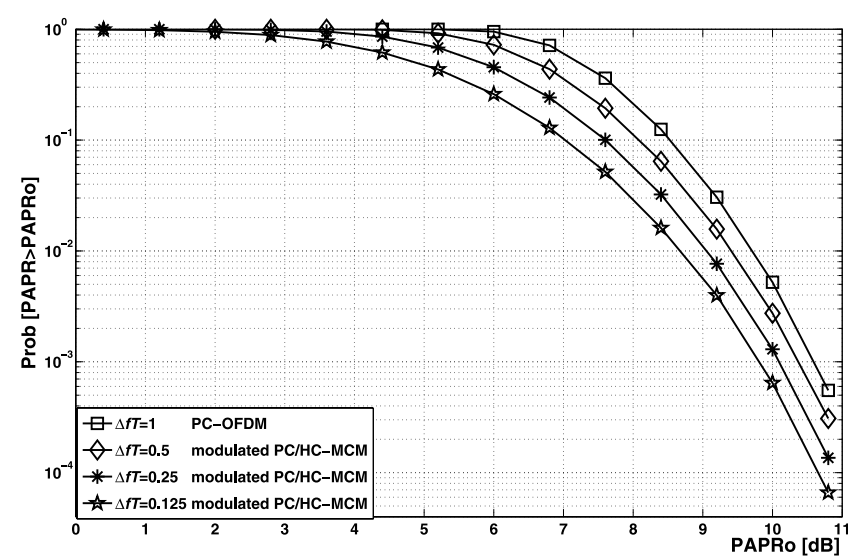

Fig. 9 CCDFs of PAPR of the modulated PC/HC-MCM with $\left(N_{C}, N_{P C}\right)$ $=(64,48)$, the modulation is QPSK.

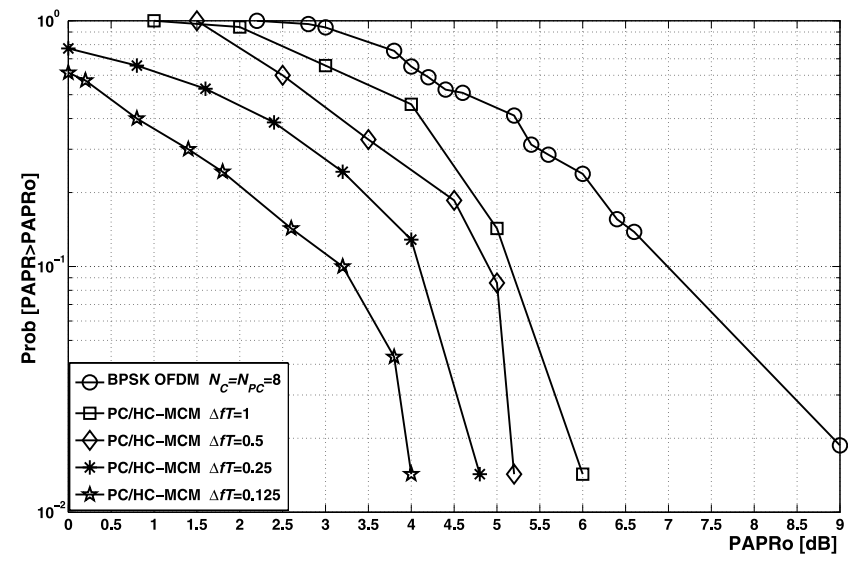

Fig. 10 CCDFs of PAPR of the PC/HC-MCM with $\left(N_{C}, N_{P C}\right)=(8,4)$ by the selection process. 


\section{PC/HC-MCM with Frequency Hopping for Multiple Access}

Multiple access (MA) in telecommunications systems refers to techniques that enable multiple users to share limited network resources efficiently. So many papers focus the attention on the MA based on OFDM [19], [20]. In this section, we develop a new frequency hopping code division MA (FH-CDMA) technique based on PC/HC-MCM.

\subsection{PC/HC-MCM with Frequency Hopping}

The PC/HC-MCM system can improve the transmitting rate if the system keeps frequency spacing $(\Delta f)$ constant for arbitrary values of modulation index $\Delta f T$. In this case, a fast transmission rate can be achieved with a smaller value of $\Delta f T$. In other words, during the same symbol duration of the PC-OFDM, the PC/HC-MCM can transmit the symbol repeatedly.

Let $T_{a}$ be the symbol duration of PC-OFDM with $\Delta f T_{a}$ $=1$ and let $T_{b}=T_{a} / 2, T_{c}=T_{a} / 4$, and $T_{d}=T_{a} / 8$ be the symbol durations of the PC/HC-MCM with $\Delta f T_{b}=0.5, \Delta f T_{c}=$ 0.25 and $\Delta f T_{d}=0.125$, respectively. Since $T_{b}, T_{c}$, and $T_{d}$ are shorter than $T_{a}$, frequency-time map for each modulation index can be illustrated as shown in Figs. 11(a)-(d). As can been seen in Figs. 11(a)-(d), during $T_{a}$, the PC/HCMCM systems can transmit two symbols for $\Delta f T_{b}$, four symbols for $\Delta f T_{c}$ and eight symbols for $\Delta f T_{d}$. Therefore, we can adopt frequency hopping (FH) technique, i.e., $L=2$ for $\Delta f T_{b}, L=4$ for $\Delta f T_{c}$ and $L=8$ for $\Delta f T_{d}$ with different length of time-slots $(L)$ to obtain the gain of $\mathrm{FH}$.

Suppose that the FH code $C=\left[C_{1}, \ldots, C_{L}\right]$, where $C_{j}(j=1, \ldots, L)$ is a $j$ th chip value that takes an integer limited over $\left[0, N_{C}-1\right]$. By the $\mathrm{FH}$ code, the input $x(k)\left(k=0, \ldots, N_{C}-1\right)$ to the IDFT in the transmitter (Fig. 2) is scrambled as $x\left(\left(k+C_{j}\right) \bmod N_{C}\right)$ for the $j$ th time-slot. The process of scrambling is also illustrated in Fig. 11. In this paper, we assume FH code whose elements (chips) are randomly generated with uniform distribution.

In the receiver (Fig. 2), the decision stage utilizes the

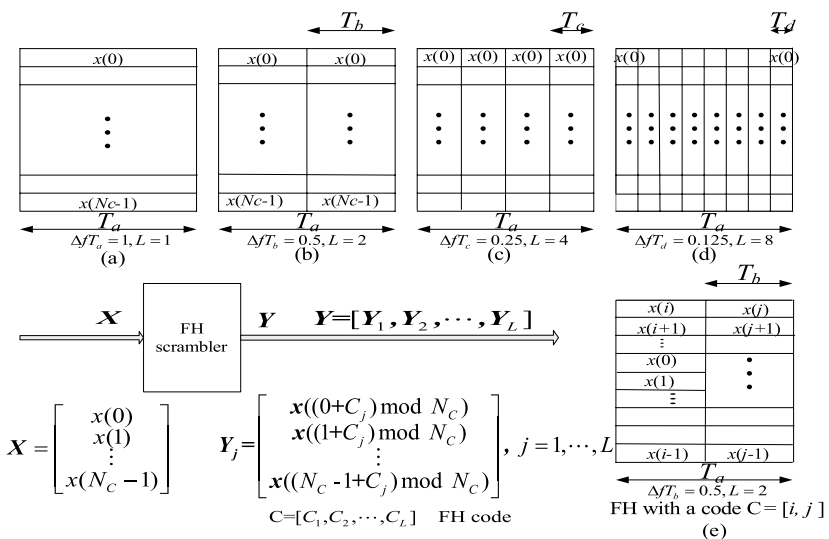

Fig. 11 Frequency hopping for the PC/HC-MCM system.
$\left(N_{C}+K_{0}\right) \times L$ input matrix to demodulate $m_{P C}$ [bits] with the MLSE in this paper.

The benefits of FH for the PC/HC-MCM can be applied in multiple access especially for downlink of system which is presented in the following subsection.

\subsection{Multiple Access for PC/HC-MCM with Frequency Hopping}

\subsubsection{System Introduction}

Figures 12(a) and (b) show a base station (BS) transmitter and a receiver for downlink of MA with $\mathrm{PC} / \mathrm{HC}-\mathrm{MCM}$ techniques. We assume that each user adopts the same $\Delta f T, L$ and combination of $\left(N_{C}, N_{P C}\right)$. The base station transmits all data of $N$ users synchronously. Input $m_{P C}$ [bits] of each user can be denoted by the matrix $\boldsymbol{Y}_{i}\left(N_{C} \times L\right)$ after the function model of FH scrambler in Fig. 11(a), and $\boldsymbol{Y}_{i}$ is processed by the IDFT column by column. After the process of $\mathrm{P} / \mathrm{S}, M_{1}$ samples $\boldsymbol{S}_{j}^{(i)}(n)\left(n=1, \ldots, M_{1}-1\right)$ of the $i$ th $(i=1, \ldots, N)$ user's signal for the $j$ th $(j=1, \ldots, L)$ time-slot can be obtained. $S_{j}^{(i)}(n)$ of all users are added to be one transmitting signal $S_{j}(t)$ after the $\mathrm{D} / \mathrm{A}$, and all users' $m_{P C}$ [bits] are transmitted in time duration of $L$ slots. Each receiver receives the transmitting signal $S_{j}(t)$ polluted by noise. After the A/D and S/P, the samples of $\hat{\mathbf{Z}}_{j}$ are obtained by the DFT of Fig. 12(b). By performing the DFT $L$ times slot by slot, we can produce a received matrix $\hat{\mathbf{Z}}=\left[\hat{\mathbf{Z}}_{1}, \ldots, \hat{\mathbf{Z}}_{L}\right]$.

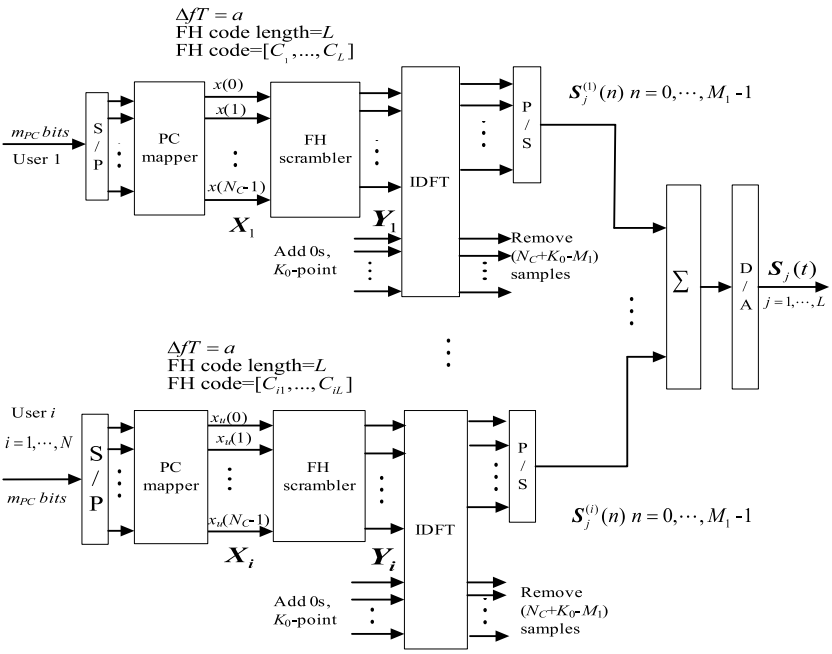

(a) Base station (BS) transmitter

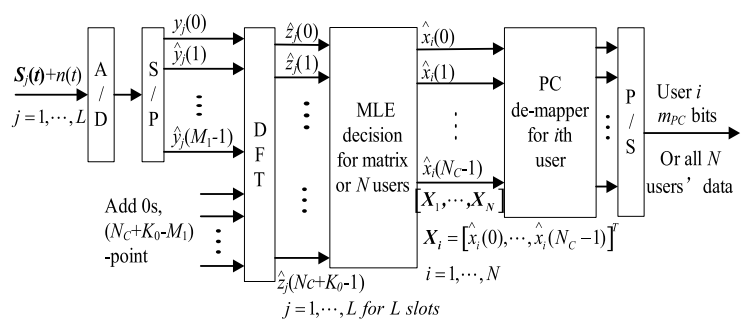

(b) Receive

Fig. 12 Downlink of MA with PC/HC-MCM techniques 
The receiver can recover all data of the users from the received matrix $\hat{\mathbf{Z}}$ based on the MLSE. The outputs of the MLSE decision stage are the estimates of the frequencies $\hat{\mathbf{X}}_{i}=\left[\hat{x}_{i}(0), \ldots, \hat{x}_{i}\left(N_{C}-1\right)\right]^{T}$ utilized for transmitting the $N_{P C}$ PC tones for the $i$ th user.

\subsubsection{System Performance}

We adopt $\left(N_{C}, N_{P C}\right)=(4,3), e^{i \theta_{i}}=1\left(i=1, \ldots, N_{P C}\right)$ for all users and $N=4$ (4 users) to explain this method. Since $\left(N_{C}, N_{P C}\right)=(4,3), m_{P C}=\left\lfloor\log _{2}\left(\begin{array}{l}4 \\ 3\end{array}\right)\right\rfloor=2$ [bits] for each user.

Figure 13 shows the minimum Euclidean distance (MED), that is, for all $m$ combinations of input data of 4 users, there will be $m$ received matrices with size of $\left(N_{C}+K_{0}\right) \times L(\hat{\mathbf{Z}})$ after the DFT of the receiver. Signal power per bit for each user is assumed to be unity. Here we make the system generate 100 different FH codes for 4 users randomly to simulate the MED with different $\Delta f T$ and $L$. All users choose the $\mathrm{FH}$ codes randomly but codes of each user are different. For each $\Delta f T$ and $L$, we use the average MED to represent the average of all 100 MEDs of the system with different FH codes. So average MED is the scale of difference between all $m$ matrices with random FH codes.

We define a new notation $T_{s}$ to represent the duration for transmitting the $m_{P C}$ data bits. Three types of selection of $L$ are shown in Fig. 13, where $T_{s}=T_{a}$ means the $m_{P C}$ data block are transmitted in one PC-OFDM symbol duration $\left(T_{a}\right)$. In this case $\Delta f T_{a}=1, L=1 ; \Delta f T_{b}=0.5, L=2$; $\Delta f T_{c}=0.25, L=4 ; \Delta f T_{d}=0.125, L=8$ can achieve the same transmission rate which is analyzed in the above section. Again, $T_{s}=2 T_{a}$ means the $m_{P C}$ data block are transmitted in two PC-OFDM symbol durations, so $\Delta f T_{a}=$ $1, L=2 ; \Delta f T_{b}=0.5, L=4 ; \Delta f T_{c}=0.25, L=8$; $\Delta f T_{d}=0.125, L=16$ generate the same transmission rate, and $\Delta f T_{a}=1, L=4 ; \Delta f T_{b}=0.5, L=8 ; \Delta f T_{c}=0.25, L=$ 16; $\Delta f T_{d}=0.125, L=32$ for $T_{s}=4 T_{a}$. Utilizing this way, $L$ can be increased with $T_{s}$.

For the case of $T_{s}=T_{a}$, the average MED and optimal MED are equal to zeros when $L=1\left(\Delta f T_{a}=1\right)$ and $L=2\left(\Delta f T_{b}=0.5\right)$ and it is no way to recover the

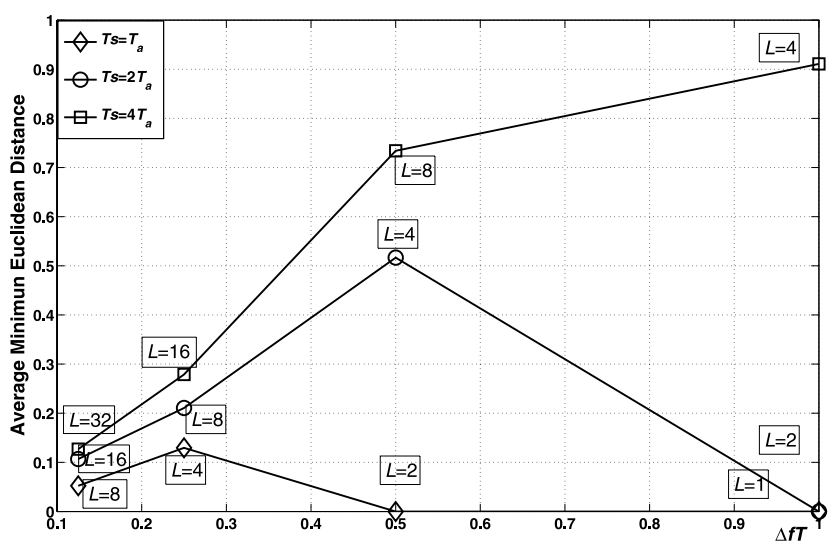

Fig. 13 Simulated minimum Euclidean distance (MED) for different frequency hopping code length $L$ with different $\Delta f T$ for 4 users. data. But with increasing $L$, such as $L=4\left(\Delta f T_{c}=0.25\right)$ and $8\left(\Delta f T_{d}=0.125\right)$, the average MED becomes larger. On the other hand, average MED increases with $T_{s}$. For $T_{s}=2 T_{a}, L=2\left(\Delta f T_{a}=1\right)$ still makes the average MED and optimal MED zero, but for $L=4\left(\Delta f T_{b}=0.5\right)$, $L=8\left(\Delta f T_{c}=0.25\right)$ and $L=16\left(\Delta f T_{b}=0.125\right)$, the average MED can be highly improved, especially for $L=$ $4\left(\Delta f T_{b}=0.5\right)$. The similar phenomena can be found with $T_{s}=4 T_{a}, L=4\left(\Delta f T_{a}=1\right)$ has the maximal average MED for all different and $L$.

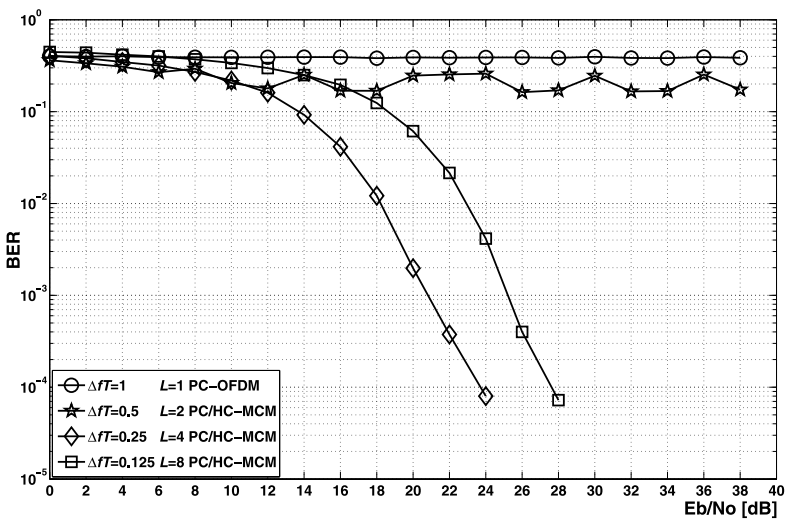

(a) $T_{s}=T_{a}$

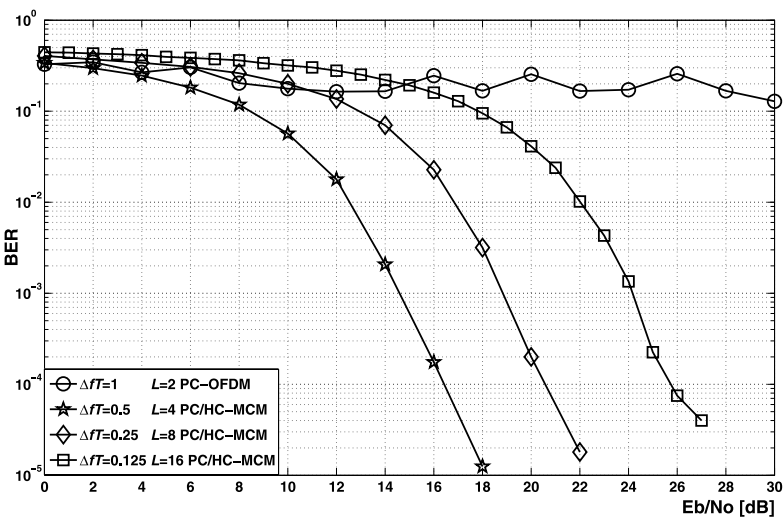

(b) $T_{s}=2 T_{a}$

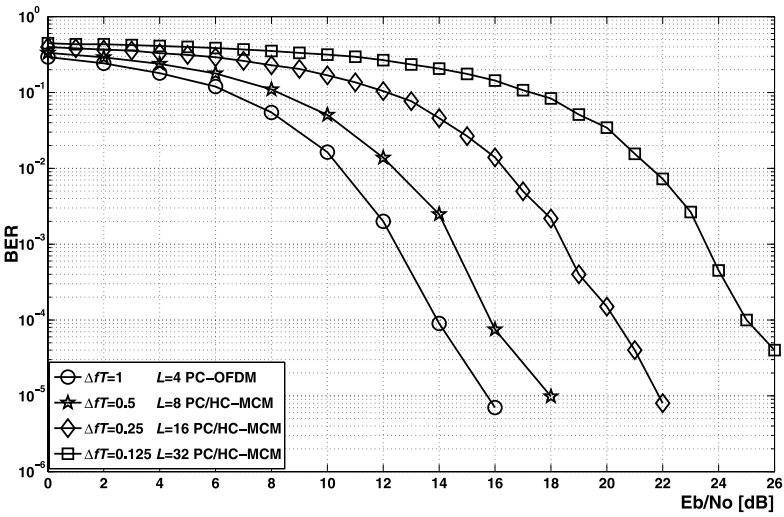

(c) $T_{s}=4 T_{a}$

Fig. 14 BER performance of FH-CDMA utilizing the PC/HC-MCM with four users over AWGN channel, $\left(N_{C}, N_{P C}\right)=(4,3), e^{j \theta_{i}}=1, i=$ $1, \ldots, N_{P C}$ and $T_{s}=1,2,4$ PC-OFDM symbol duration $\left(T_{a}\right)$ for different $L$ with optimal FH codes. 
The distribution of MED varies with $\Delta f T$ and $L$. For the case of $T_{s}=4 T_{a}$ and $L=4\left(\Delta f T_{a}=1\right)$, we make the system generate 100 different $\mathrm{FH}$ codes combination for 4 users randomly to simulate the MED, and the MEDs of 21 combinations are zeros. But for other $\Delta f T$, especially for $\Delta f T_{c, d}=0.25,0.125$, the above phenomena never happens. So it is very important for the system to adopt the appropriate $\mathrm{FH}$ codes and $\Delta f T$.

Figures 14(a)-(c) show the simulated results of the BER performance with 4 users over the AWGN channel. Note that here we make each user choose the FH codes with the optimal MED from all simulated FH codes. From Fig. 13(a), we can find that with $T_{s}=T_{a}, L=1$ and 2, the BER performance vibrate between 0.5 and 0.1 , so no any data can be recovered from the received matrices with white noise. But when the system adopts $L=4\left(\Delta f T_{c}=0.25\right)$ and $L=8\left(\Delta f T_{d}=0.125\right)$, the data of users can be recovered. It also means that the system can support 4 users to transmit synchronously in one symbol duration of PCOFDM when system adopts PC/HC-MCM. For $T_{s}=2 T_{a}$, $L=8\left(\Delta f T_{b}=0.5\right)$ can achieve the best BER performance, and for the $T_{s}=4 T_{a}, L=4\left(\Delta f T_{a}=1\right)$ can also get the optimal performance.

Figures 14(a)-(c) also show that the system can change its transmission rate to improve the BER performance over the AWGN channel. For $T_{s}=T_{a}$, if the system chooses $L=$ $4\left(\Delta f T_{c}=0.25\right)$ to hold 4 users at BER $=10^{-4}$, the required $\mathrm{Eb} / \mathrm{No} \simeq 23.9[\mathrm{~dB}]$. But for $T_{s}=2 T_{a}$, if the system chooses $L=8\left(\Delta f T_{c}=0.25\right)$ to hold 4 users, the required $\mathrm{Eb} / \mathrm{No}$ reduces to $20.6[\mathrm{~dB}]$, but the transmission rate for $T_{s}=2 T_{a}$ is half of that for system with $T_{s}=T_{a}$.

\section{Discussions and Conclusions}

OFDM symbols and systems, in a historical and practical perspective, can be viewed and analyzed as the system with $\Delta f_{0} T_{0}=1$ or $\Delta f_{0}\left(T_{0}+T g\right)>1$. To our knowledge, it is first time for us to focus attentions on the case that $\Delta f T$ can be extended to 0.125 for parallel combinatory multi-carrier systems.

In this paper, we proposed two types of $\mathrm{PC} / \mathrm{HC}-\mathrm{MCM}$ systems, which are named as modulated PC/HC-MCM system and PC/HC-MCM system. The modulated PC/HC$\mathrm{MCM}$ system can achieve better BER performance than that of the HC-MCM with the equal BWE. The PC/HC-MCM system can obtain excellent PAPR by selecting the suitable constellations. Furthermore, we also combined the PC/HCMCM and the FHMA to transmit multiple users' data synchronously within one symbol duration of PC-OFDM.

The MLSE has the high complexity of demodulation, therefore, it limits the PC/HC-MCM with smaller $N_{C}$. Although we have presented an algorithm to reduce the complexity [14], the BER performance is decreased especially with the smaller $\Delta f T$. Furthermore, the PC/HC-MCM system designs the better PAPR by computer search, therefore, high computation complexity also increases especially for large $\left(N_{C}, N_{P C}\right)$. On the other hand, the number of users which can be held in the MA system with PC/HC-MCM is limited and depends on the combination of $\left(N_{C}, N_{P C}\right), \Delta f T$ and sets of FH codes. Those issues will be presented in other papers.

\section{References}

[1] M. Burgos-Garcia and F. Perez-Martinez, "Simple procedure for optimum linearization of amplifiers in multicarrier application," Electron. Lett., vol.30, no.2, pp.114-115, Jan. 1994.

[2] Y. Sun, "Bandwidth-efficient of wireless OFDM,' IEEE J. Sel. Areas Commun., vol.19, no.11, pp.2267-2278, Nov. 2001

[3] R.V. Nee and R. Prasad, OFDM for Wireless Multimedia Communications, Artech House, 2000.

[4] J. Zhu, S. Sasaki, and G. Marubayashi, "Proposal of parallel combinatory spread spectrum communication system," IEICE Trans. Commun. (Japanese Edition), vol.J74-B, no.5, pp.207-214, May 1991

[5] S. Sasaki, H. Kikuchi, J. Zhu, and G. Marubayashi, "Multiple access performance of parallel combinatory spread spectrum commmunication systems in nonfading and Rayleigh fading channels," IEICE Trans. Commun., vol.E78-B, no.8, pp.1152-1161. Aug. 1991.

[6] P.K. Frenger, N. Arne, and B. Svensson, "Parallel combinatory OFDM signaling," IEEE Trans. Commun., vol.47, no.4, pp.558565, April 1999.

[7] G. Kutyniok and T. Strohmer, "Wilson bases for general timefrequency lattices," SIAM J. Math Anal., vol.37, no.3, pp.685-7111, 2005.

[8] T. Strohmer and S. Beaver, "Optimal OFDM design for timefrequency dispersive channels," IEEE Trans. Commun., vol.51, no.7, pp.1111-1122, July 2003.

[9] J.R. Treichler, M.G. Larimore, and J.C. Harp, "Practical blind demodulator for high-order QAM signals," Proc. IEEE, vol.86, no.3, pp.1907-1926, Oct. 1998.

[10] N. Morinaga, M. Yokoyama, and S. Sampei, "Intelligent radio communications techniques for advanced wireless communication systems," IEICE Trans. Commun., vol.E79-B, no.3, pp.214-221, March 1996.

[11] M. Hamamura and S. Tachikawa, "Bandwidth efficiency improvement for multi-carrier systems," Proc. IEEE PIMRC2004, pp.48-52, Barcelona, Spain, Sept. 2004.

[12] M. Hamamura and S. Tachikawa, "On high compaction FDM comunication systems," IEICE Technical Report, WBS2003-45, July 2003.

[13] Y. Hou and M. Hamamura, "Bandwidth efficiency of PC-OFDM systems with high compaction multi-carrier modulation," Proc. IEEE WCNM2005, pp.177-180, Wuhan, China, Sept. 2005.

[14] S. Takahashi, M. Hamamura, and S. Tachikawa, "A demodulation complexity reduction method using $M$-algorithm for high compaction multi-carrier modulation systems," Proc. IEEE ISWCS2004, pp.336-341, Mauritius, Sept. 2004.

[15] B. Muquet, Z. Wang, G.B. Giannakis, M. de Courville, and P. Duhamel, "Cyclic prefixing or zero padding for wireless multicarrier transmissions?," IEEE Trans. Commun., vol.50, no.12, pp.21362148, Dec. 2002

[16] K. Halford and M. Webster, "Multipath measurement in wireless LANs," Intersil Application Note AN9895, Oct. 2001.

[17] B. Tarokh and H.R. Sadjadpour, "Construction of M-QAM signals utilizing QPSK Golay sequences with low PMEPR suitable for OFDM systems," IEEE Trans. Commun., vol.51, no.1, pp.25-28, Jan. 2003.

[18] C. Tellambura, "Computation of the continuous-time PAR of an OFDM signal with BPSK subcarriers," IEEE Commun. Lett., vol.5, no.5, pp.185-187, May 2001.

[19] W.C.Y. Lee, "CS-OFDMA: A new wireless CDD physical layer scheme," IEEE Commun. Mag., vol.43, no.2, pp.74-79, Feb. 2005. 
[20] A. Jamalipour, T. Wada, and T. Yamazato, "A tutorial on multiple access technologies for beyond 3G mobile networks," IEEE Commun. Mag, vol.43, no.2, pp.110-117, Feb. 2005.

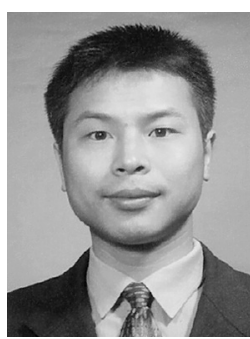

Yafei Hou received his B.S. degree in Electronic Engineering from Anhui University of Technology and Science, China, in 1999 and M.S. degree in Computer Science from Wuhan University, China, in 2002. During 2002-2004, as a Ph.D. candidate he was at Department of Computing and Information Technology, Fudan University, China. He is now a Ph.D. candidate at Kochi University of Technology (KUT), Japan, as an international student of cooperation between KUT and Fudan University. His main areas of interest are communication systems, wireless networks, ad-hoc networks and signal processing.

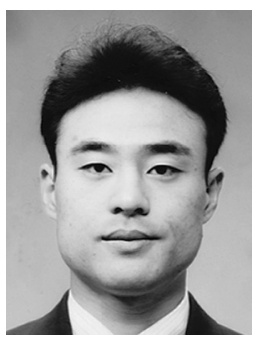

Masanori Hamamura received his B.S., M.S. and Ph.D. degrees in electrical engineering from Nagaoka University of Technology, Nagaoka, Japan, in 1993, 1995 and 1998, respectively. From 1998 to 2000, he was a Research Fellow of the Japan Society for the Promotion of Science. Since 2000, he has been with the Department of Information Systems Engineering at Kochi University of Technology, Kochi, Japan, where he is now an Associate Professor. From 1998 to 1999 , he was a visiting researcher at Centre for Telecommunications Research, King's College, London, United Kingdom, where he worked on adaptive signal processing for mobile systems. His current research interests are in the areas of spread spectrum systems, mobile communications and signal processing. Dr. Hamamura is a member of IEEE. 